\title{
Racial and ethnic differences in atrial fibrillation risk factors and predictors in women: Findings from the Women's Health Initiative
}

Fatima Rodriguez, MD, MPH, ${ }^{a}$ Marcia L. Stefanick, PhD, ${ }^{b}$ Philip Greenland, MD, ${ }^{\mathrm{c}}$ Elsayed Z. Soliman, MD, ${ }^{\mathrm{d}}$ JoAnn E. Manson, MD, DrPH, ${ }^{\mathrm{e}}$ Nisha Parikh, MD, MPH, ${ }^{\mathrm{f}}$ Lisa W. Martin, MD, ${ }^{\mathrm{g}} \mathrm{Joseph}^{\mathrm{C} .}$ Larson, MS, ${ }^{\mathrm{h}}$ Mark Hlatky, MD, ${ }^{\mathrm{a}}$ Rami Nassir, PhD, ${ }^{\mathrm{i}}$ Crystal W. Cené, MD, MPH, ${ }^{\mathbf{j}}$ Beatriz L. Rodriguez, MD, PhD, ${ }^{\mathrm{k}}$ Christine Albert, MD, MPH, ${ }^{1}$ and Marco V. Perez, MD a Stanford, CA; Chicago, IL; Winston Salem, NC; Boston, MA; San Francisco, CA; Washington, DC; Seattle, WA; Davis, CA; Chapel Hill, NC; and Honolulu, HI

Background The incidence of atrial fibrillation (AF) is higher in non-Hispanic whites (NHWs) compared with other raceethnic groups, despite more favorable cardiovascular risk profiles. To explore reasons for this paradox, we compared the hazards of AF from traditional and other risk factors between 4 raceethnic groups in a large cohort of postmenopausal women.

Methods We included 114,083 NHWs, 11,876 African Americans, 5,174 Hispanics, and 3,803 Asians from the Women's Health Initiative free of AF at baseline. Women, averaging 63 years old, were followed up for incident AF using hospitalization records and diagnostic codes from Medicare claims.

Results Over a mean of 13.7 years, 19,712 incident cases of AF were recorded. Despite a higher burden of hypertension, diabetes, and obesity, annual AF incidence was lower among nonwhites $10.7 \%, 0.4 \%$, and $0.4 \%$ for African American, Hispanic, and Asian participants, respectively, compared with $1.2 \%$ for NHWs). The hazards of AF from hypertension, diabetes, obesity, heart failure, and coronary artery disease were similar across raceethnic groups. Major risk factors, including hypertension, obesity, diabetes, smoking, peripheral arterial disease, coronary artery disease, and heart failure, accounted for an attributable risk of $50.3 \%$ in NHWs, $83.1 \%$ in African Americans, $65.6 \%$ in Hispanics, and $37.4 \%$ in Asians. Established AF prediction models performed comparably across raceethnic groups.

Conclusions In this large study of postmenopausal women, traditional cardiovascular risk factors conferred a similar degree of individual risk of AF among 4 race-ethnic groups. However, major AF risk factors conferred a higher-attributable risk in African Americans and Hispanics compared with NHWs and Asians. (Am Heart J 2016;176:70-7.)

\section{Background}

Atrial fibrillation (AF) is the most common cardiac arrhythmia and confers a significant risk of morbidity and mortality. ${ }^{1,2}$ It accounts for 75,000 to 100,000 strokes

From the ${ }^{a}$ Division of Cardiovascular Medicine, Stanford University, Stanford, CA, ${ }^{b}$ Department ofMedicine, Stanford Prevention Research Center, Stanford University, Stanford, CA, ${ }^{C}$ Department of Preventive Medicine, Northwestern School of Medicine, Chicago, IL, ${ }^{d}$ Epidemiological Cardiology Research Center (EPICARE), Wake Forest School of Medicine, Winston Salem, NC, 'Division of Preventive Medicine, Department of Medicine, Brigham and Women's Hospital and Harvard Medical School, Boston, MA, 'Division of Cardiovascular Medicine, University of California San Francisco School ofMedicine, San Francisco, CA, ${ }^{9}$ Division of Cardiology, George Washington University School of Medicine and Health Sciences, Washington, DC, hata Coordinating Center, Fred Hutchinson Cancer Research Center, Seattle, WA, 'Department of Biochemistry and Molecular Medicine, University of California Davis, Davis, CA, 'Division of General Internal Medicine, University of North Carolina at Chapel Hill, Chapel Hill, NC, ${ }^{k}$ Divison of Geriatrics, University of Hawaii at Manoa, Honolulu, HI, and 'Division of Cardiology, Brigham and Women's Hospital, Harvard Medical School, Boston, MA.

Submitted February 8, 2016; accepted March 8, 2016.

Reprint requests: Fatima Rodriguez, MD, MPH, Division of Cardiovascular Medicine, Stanford University, 870 Quarry Road, Falk CVRC, Stanford, CA 94305-5406.

E-mail: frodrigu@stanford.edu

0002-8703

http://dx.doi.org/10.1016/i.ahj.2016.03.004 per year, approximately $15 \%$ of all strokes in the United States. ${ }^{3}$ It is associated with increased mortality after adjustment for numerous cardiovascular risk factors and comorbidities, ${ }^{4}$ and has been linked to increased risk of myocardial infarction ${ }^{5}$ and sudden cardiac death. ${ }^{6}$ Women comprise $60 \%$ of individuals with AF older than 75 years, and the clinical consequences of AF for women are substantial. ${ }^{7}$ Women with $\mathrm{AF}$ are at higher risk for stroke and myocardial infarction, and have a higher overall mortality risk than do men. ${ }^{3,4,8}$

Analyses of large prospective cohorts, including the Framingham Heart Study, ${ }^{9}$ the Cardiovascular Health Study ${ }^{10}$ and others, ${ }^{11-13}$ have established principal independent risk factors of AF including age, hypertension, diabetes mellitus, obesity, myocardial infarction, coronary heart disease, and heart failure. However, these prior studies were conducted in primarily non-Hispanic white (NHW) populations, potentially limiting their generalizability to other race-ethnic groups. ${ }^{14}$

Rates of AF vary significantly between different racial and ethnic groups. ${ }^{1,15}$ The incidence of AF is lower in 
African Americans compared with NHWs in the Women's Health Initiative Observational Study (WHI OS), ${ }^{16}$ the Cardiovascular Health Care Study, ${ }^{10}$ and several other study cohorts, independent of socioeconomic and other risk factors. ${ }^{15,2,17,18}$ Hispanics and Asians also have lower rates of AF compared with NHWs. ${ }^{15,16,19}$

The difference in rates of AF among race-ethnic groups may be explained by either differences in underlying genetic susceptibility, differences in prevalence of risk factors of $\mathrm{AF}$, differences in the hazards due to individual risk factors, or a combination of these variables. Although a portion of the race-ethnic difference may be explained by genetic ancestry in some populations, ${ }^{20}$ this was not the case in older women in the WHI. ${ }^{21}$ We therefore sought to characterize the differences in the hazards due to several risk predictors for AF by race and ethnicity in the WHI, to measure differences in the attributable risk (AR) due to established AF risk factors, and to validate existing AF predictive models in this well-characterized and diverse population of postmenopausal women.

\section{Methods}

\section{Study population}

In order to maximize the number of participants from the different race-ethnic groups, the study population included all NHW, African American, Hispanic, and Asian participants from the WHI OS and the randomized clinical trials (RCTs). Participants who self-identified as "Other" were excluded from these analyses. The methods of WHI recruitment and follow-up are described elsewhere in detail. ${ }^{22-24}$ Briefly, 93,676 postmenopausal women were enrolled in the WHI OS and 68,132 women were enrolled in at least one of the RCTs of hormone therapy, dietary modification, and calcium/vitamin D, between October 1993 and December 1998. Participants were recruited primarily through mass mailings and enrolled at 1 of 40 US clinical centers. Participants were postmenopausal aged between 50 and 79 years at the time of study enrollment. After initial study enrollment, participants were under continued surveillance for cardiovascular disease outcomes, including new-onset AF. The average time of follow-up was 13.7 years extending through 2014. Participants with AF at baseline (as reported on the initial questionnaire or evidenced on baseline electrocardiogram [ECG]) or with missing covariate data were excluded from these analyses.

\section{Baseline variables}

Participants completed self-administered questionnaires at study enrollment, including demographic and clinical characteristics at baseline. Study questionnaires, physical measurements, and quality assurance have been detailed previously. ${ }^{22,23}$ Race and ethnicity were self-reported in the baseline questionnaire. Participants underwent measurement of blood pressure, height, weight, and hip and waist circumferences at enrollment. Body mass index (BMI) was calculated as weight (in kilograms) divided by the square of measured height (in meters squared). Age, income, education, history of hypertension, diabetes, hyperlipidemia, coronary artery disease (CAD), stroke, congestive heart failure (CHF), peripheral artery disease (PAD), smoking, and alcohol use were ascertained by self-report on baseline questionnaires. Participants with measured resting systolic blood pressure $\geq 140 \mathrm{~mm} \mathrm{Hg}$ or diastolic blood pressure $\geq 90$ $\mathrm{mm} \mathrm{Hg}$ at the initial clinic visit were also classified as hypertensive. Physical activity was assessed using selfreported questionnaires and frequency, intensity, duration, and types of physical activity were evaluated as previously described. ${ }^{25,26}$ Standard resting 12-lead ECGs (MAC PC; Marquette Electronics Inc, Milwaukee, WI) were recorded in participants enrolled in the RCTs and processed using the 2001 version of the GE Marquette 12-SL program (GE Marquette Electronics, Inc) in a central electrocardiography laboratory. The PR interval was determined as the time between the onset of the p-wave and the onset of the QRS complex.

\section{Ascertainment of incident $\mathrm{AF}$}

Identification of cases of incident AF has been described in previous studies. ${ }^{16,27}$ Participants completed annual questionnaires updating their interval medical history. Medical records were obtained in the event of self-reported hospitalization and the International Classification of Diseases, Ninth Revision (ICD-9) code for AF (427.31) was extracted from these records. WHI data were also linked with Centers for Medicare \& Medicaid Services (CMS) data using social security numbers, birth dates, and death dates, with $97 \%$ of Medicare-eligible WHI participants successfully linked. Among those with Medicare coverage, incident AF was identified by the first occurrence of ICD-9 code 427.31 in any diagnosis position in the inpatient (MEDPAR), outpatient, and carrier files during the years 1994 to 2012. Several sensitivity analyses were performed with atrial flutter (ICD-9 code 427.32).

Because CMS data were available for some participants, but not others, at different periods over WHI follow-up, a time-dependent indicator variable of Medicare coverage was added to the Cox hazard models described below to adjust for possible ascertainment bias related to differential exposure to CMS. Medicare time eligible for analysis included those intervals where participants were enrolled in fee-for-service Medicare and not simultaneously enrolled in a Medicare-managed care plan.

\section{Statistical analysis}

Baseline characteristics of participants were compared across race-ethnic categories, using either analysis of variance for continuous variables or the $x^{2}$ test for categorical variables. 
Table I. Baseline characteristics of study population by race-ethnic groups

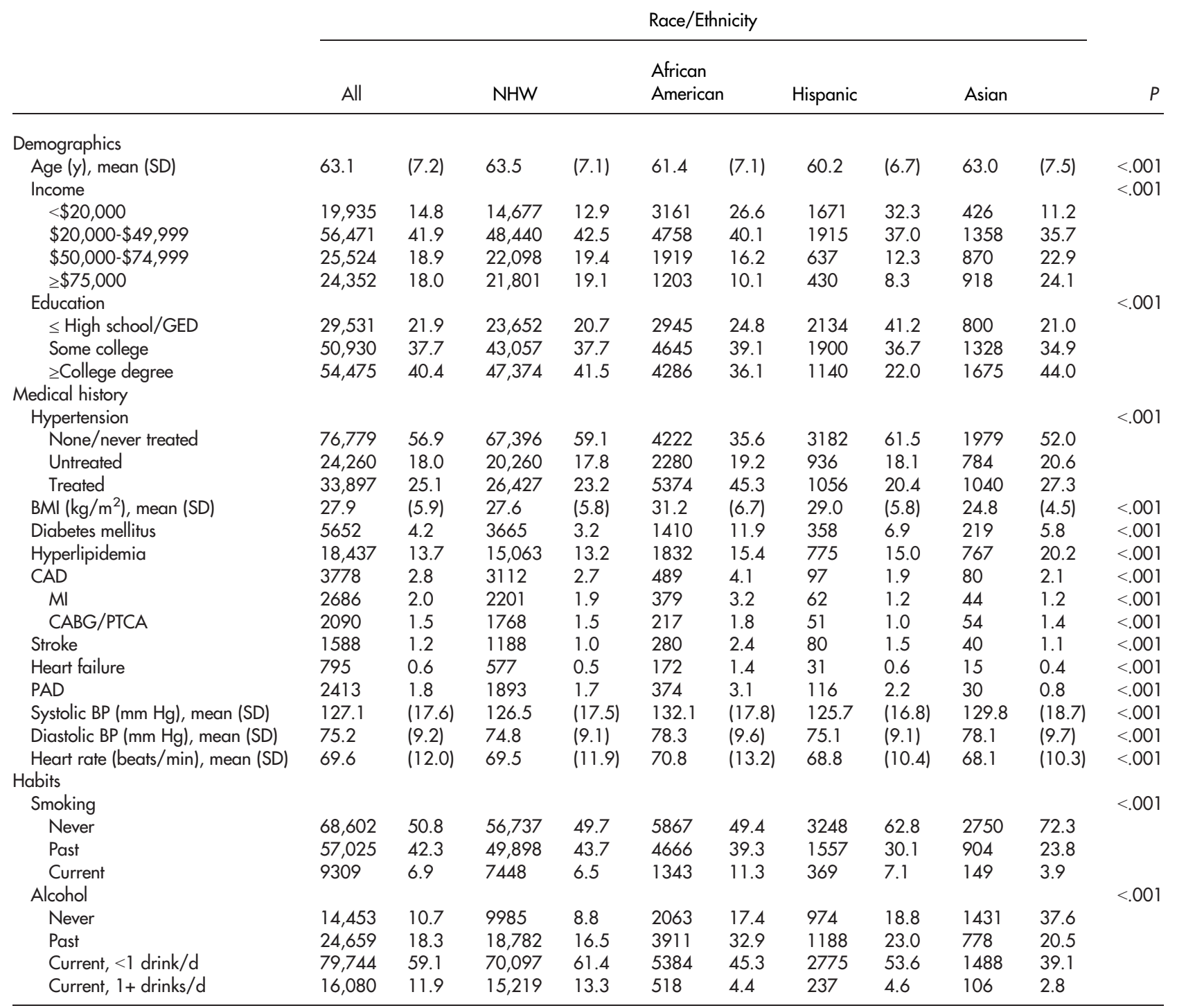

Abbreviations: $M I$, myocardial infarction; $C A B G$, coronary artery bypass grafting; PTCA, percutaneous transluminal coronary angioplasty; BP, blood pressure.

We calculated the AR for each of the preventable or treatable risk factors for $\mathrm{AF}$ in each of the different race-ethnic cohorts. The AR was calculated as the difference between the rate of $\mathrm{AF}$ in the entire racial-ethnic cohort minus the rate of $\mathrm{AF}$ in the race-ethnic cohort without the risk factor, divided by the rate of $\mathrm{AF}$ in the entire racial-ethnic cohort, multiplied by 100 .

The relationship between risk factors and incident AF was examined using the multivariate Cox regression models for each of the different race-ethnic groups. Multivariate analyses were performed using a primary model containing the following covariates with known or suspected association with AF: age, education, BMI, hypertension, diabetes, CAD, CHF, PAD smoking, and alcohol use. The associations between physical activity, height, PR interval, and incident AF were assessed using a multivariate Cox regression model adjusted for all covariates in the primary regression model. All regression models were additionally adjusted for time-dependent exposure to Medicare coverage, as has been done in prior analyses, ${ }^{16}$ as well as inclusion in a WHI randomized trial and WHI intervention assignment. To determine if AF risk conferred by the different risk factors differed across different race-ethnic groups, we measured the interaction between race/ethnicity and each of the covariates by including a risk factor* race/ethnicity term in the primary multivariate Cox regression model. Associations between risk factors and AF were reported as hazard ratios (HRs) with $95 \%$ CIs. The proportional hazards assumption was 


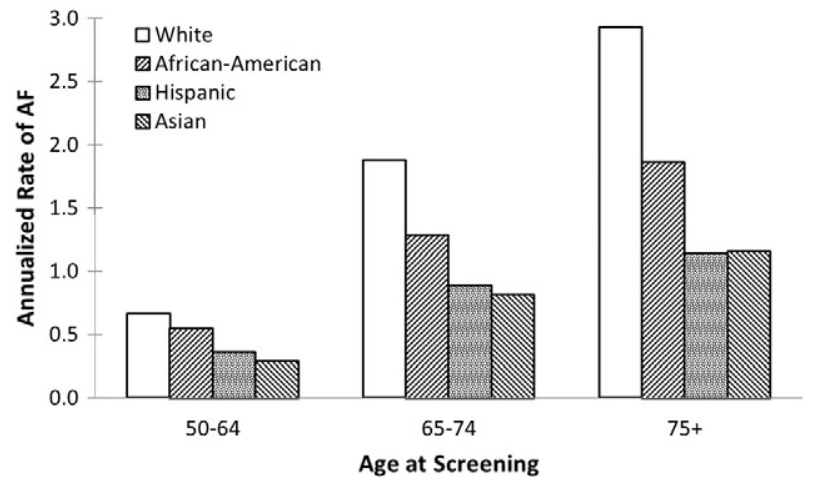

Annualized rates of AF by age and race.

verified by visual inspection of the log-likelihood plots of developing AF over time.

Finally, the performance of the Framingham Heart Study AF Score, ${ }^{28}$ Atherosclerosis Risk in Communities Study (ARIC), ${ }^{29}$ and Women's Health Study (WHS) ${ }^{30}$ regression models was tested at 5 and 10 years by estimating the $c$ statistic for these models in each of the different race-ethnic cohorts. Because only the subset of women from the RCTs had ECG data available, a $c$ statistic was calculated both for the full cohort without PR interval in the model, as well as for the subgroup of women with ECG data $(n=50,428)$ with the PR interval measurement.

Analyses were performed using SAS statistical software (version 9.4; SAS Institute Inc, Cary, NC). The WHI program is funded by the National Heart, Lung, and Blood Institute, National Institutes of Health, US Department of Health and Human Services through contracts HHSN268201100046C, HHSN268201100001C, HHSN268201100002C, HHSN268201100003C, HHSN268201100004C, and HHSN271201100004C. Dr Marco Perez is funded by the Harold Amos Faculty Development Program Award from the Robert Wood Johnson Foundation and a Fellow to Faculty Award from the American Heart Association.

The authors are solely responsible for the design and conduct of this study, all study analyses, the drafting and editing of the manuscript, and its final contents.

\section{Results}

\section{Baseline characteristics}

Of the 158,833 NHW, African American, Hispanic, and Asian women enrolled in the WHI OS and RCTs, 6,926 were excluded because of baseline AF, 631 were excluded for lack of follow-up, and 16,971 were excluded due to missing covariate data of interest. There were a total of 114,083 NHW, 11,876 African American, 5,174
Hispanic, and 3,803 Asian women who met the inclusion criteria. Baseline characteristics of the participants are summarized in Table I. On average, women were 63.1 years of age, and most (78.1\%) had some college education or higher. African American and Hispanic women were younger and had lower annual incomes. More than $40 \%$ of Hispanic participants had a high school education or less as compared with $21 \%$ of NHW women, $25 \%$ of African American women, and 21\% of Asian women $(P<.001)$.

The prevalence of all traditional AF risk factors, including hypertension, BMI, diabetes, CAD, CHF, and PAD, differed across race-ethnic groups (analysis of variance $P<.001$ for each risk factor, Table I). Notably, almost two-thirds of the African American women in the study had hypertension in contrast to only $41 \%$ of NHW women. African American and Hispanic women had higher BMIs and were more likely to have diabetes compared with NHW women. Certain cardiovascular diseases, including $\mathrm{CAD}, \mathrm{CHF}$, and $\mathrm{PAD}$, were more prevalent in African American women compared with NHW women (Table I). Asian women had on average a lower BMI than did NHW women $\left(24.8 \mathrm{vs} 27.6 \mathrm{~kg} / \mathrm{m}^{2}\right)$, but higher rate of diabetes (5.8\% vs $3.2 \%$ ).

\section{Risk factors for AF by race-ethnic subgroups}

There were 18,090, 1,097, 295, and 233 incident AF cases over an average of 13.7 years of follow-up among NHW, African American, Hispanic, and Asian women, respectively. The annualized rates of AF by age and race/ ethnicity are shown in Figure 1. Non-Hispanic white women consistently had higher incidence of AF among all age groups. Hispanic and Asian women had the lowest rates of $\mathrm{AF}$ in all age groups. These differences were more pronounced in the older age groups. There were a total of 3,895 incident atrial flutter cases during follow-up.

The impact of traditional and other AF risk factors on $\mathrm{AF}$ incidence by race/ethnicity after multivariate 
Figure 2

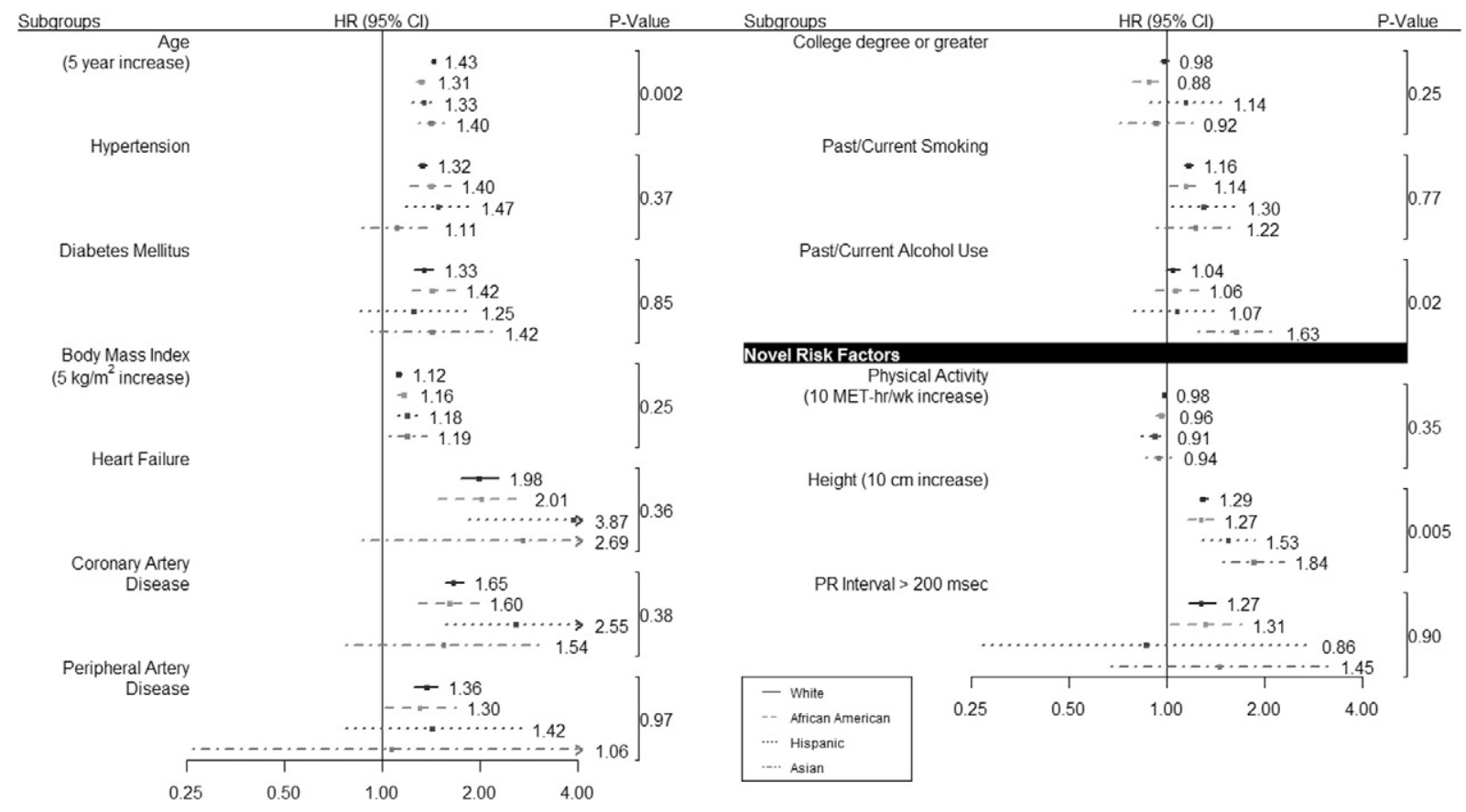

Multivariate-adjusted HRs of traditional and novel AF risk factors by race/ethnicity. ' HRs and interaction $P$ values from a proportional hazards model with the risk factor of interest, race/ethnicity, and their interaction, and are stratified within the model by WHI intervention arms, WHI extension 2 membership, and time-dependent fee-for-service Medicare coverage. Models are additionally adjusted for education, pills for high cholesterol, WHI intervention assignments, and all traditional risk factors in Table II.

adjustment is shown in Figure 2. The hazards of AF due to age were slightly higher in NHW and Asians, compared with African Americans and Hispanics (HR for a 5-year increase $1.43,1.40$ vs 1.31 and 1.33 , respectively; $P$ interaction $=.002$ ). Hazard ratios for the traditional risk factors of hypertension, diabetes, BMI, and heart failure for incident AF were similar in NHW, African American, Hispanic, and Asian women. Although the HRs for AF due to hypertension, CHF, and CAD were higher in Hispanics compared with NHW, there was no statistically significant interaction across all race-ethnic groups. Alcohol use was associated with an increased risk of AF to a greater degree in Asians compared with NHW, African Americans, and Hispanics (HR 1.65 vs $1.04,1.06,1.06$, respectively; $P$ interaction $=.02$ ).

The multivariate-adjusted HRs of novel AF risk factors by race/ethnicity are shown in Figure 2 . Height was a significant predictor of AF for all race-ethnic groups with a higher predictive value among Hispanics and Asians compared with NHW and African Americans (HR for every $10-\mathrm{cm}$ increase $1.53,1.82$ vs 1.28 and 1.27 , respectively; $P$ interaction $=.007$ ).

\section{Attributable risk}

Table II demonstrates the ARs for reversible or treatable $\mathrm{AF}$ risk factors by race/ethnicity. The AR due to hypertension was highest in the African American women (39.0\%) and lowest in Asian women (18.3\%). More than 83\% of the AR for African American women and 65.6\% for Hispanic women are accounted for by modifiable risk factors for AF, as compared with just $50.3 \%$ for NHW women and $37.4 \%$ for Asian women. Alcohol consumption was not included because NHW, African American, and Hispanic women who used alcohol had a lower rate of AF compared with nonusers; however, alcohol accounted for $15 \%$ of the AR in Asian women (data not shown), second only to hypertension in that group.

Similarly, stroke accounted for a small fraction of the AR for all populations: $0.8 \%$ for NHWs, $1.0 \%$ for African Americans, $1.8 \%$ for Hispanics, and $1.7 \%$ for Asians. Stroke was not included in Table II because it is not generally considered a risk factor for AF since previously unidentified silent forms of AF may account for some of the strokes.

\section{Atrial fibrillation prediction models}

In the overall cohort, the Framingham, ARIC, and WHS AF prediction models and the primary WHI regression model used in this study performed similarly well in all race-ethnic groups for both 5-and 10-year risk predictions ( $c$ statistic 0.78-0.81) (Table III). Each of the models also performed as well or slightly better in NHW race-ethnic groups compared with the NHW group. In the WHI 
Table II. Attributable risk (\%) of traditional AF risk factors

\begin{tabular}{|c|c|c|c|c|c|}
\hline Characteristic & Level of interest & $\mathrm{NHW}$ & African American & Hispanic & Asian \\
\hline Hypertension & Yes & 24.3 & 38.3 & 29.2 & 18.3 \\
\hline $\mathrm{BMI}$ & $>25 \mathrm{~kg} / \mathrm{m}^{2}$ & 13.1 & 18.9 & 15.7 & 6.9 \\
\hline Diabetes & Yes & 2.6 & 8.7 & 3.6 & 4.9 \\
\hline Smoking & Past/Current & 4.3 & 5.4 & 9.1 & 4.2 \\
\hline PAD & Yes & 1.6 & 3.4 & 1.6 & 0.2 \\
\hline Heart failure & Yes & 0.9 & 2.9 & 2.0 & 1.1 \\
\hline Coronary heart disease & Yes & 3.5 & 5.5 & 4.4 & 1.8 \\
\hline Total & & 50.3 & 83.1 & 65.6 & 37.4 \\
\hline
\end{tabular}

regression model, the $c$ statistics for 10-year prediction in the Asian (0.87, 95\% CI 0.83-0.90), Hispanic (0.85, 95\% CI 0.82-0.87), and African American (0.83, 95\% CI 0.82-0.85) groups were higher than that in NHW $(0.81,95 \%$ CI 0.80-0.81), although the CIs overlap.

\section{Discussion}

In long-term follow-up of a large, diverse cohort of postmenopausal women, we found that although minority women have a lower incidence of AF, many traditional risk factors are more prevalent in some race-ethnic cohorts, but individually contribute to AF risk to a similar extent compared with NHWs. However, at the population level, these risk factors contribute more to AF risk in African Americans and Hispanics compared with NHW and Asian counterparts. Importantly, particularly for African American women, most of the AF risk may be attributable to modifiable or treatable risk factors. We also found that AF risk prediction models perform similarly well in all race-ethnic groups.

The reasons for the observed racial and ethnic differences in AF incidence and risk factors are not well understood. Paradoxically, African Americans and Hispanics typically have higher rates of obesity, hypertension, and other cardiovascular risk factors, but a lower rate of $\mathrm{AF}{ }^{1,31}$ Disparities in socioeconomic status and lack of exposure to the health care system leading to underreporting and ascertainment of $\mathrm{AF}$ in underrepresented minority groups have been implicated for these differences. ${ }^{32}$ However, the relationship between race and AF persists after adjusting for a wide range of socioeconomic and other risk factors, with African Americans typically having a lower prevalence of AF. ${ }^{33,34}$ This finding is seen even in veteran populations with similar access to health care and in clinical subgroups already receiving medical care, such as those with heart failure. ${ }^{35}$ Atrial fibrillation is also detected at a lower rate among African Americans who are constantly monitored with implanted cardiac devices. ${ }^{36}$

One potential explanation for these racial-ethnic disparities is a difference in genetic susceptibility to AF. Although this may be true in younger populations, ${ }^{20}$ we did not find this to be the case in the admixture study of the WHI SHARe African American cohort, which included older women. ${ }^{21}$ This suggests that, at least in older women, clinical and environmental factors are more likely to account for the observed differences. We found that, particularly among African Americans, most of the attributable risk of AF can be explained by traditional, reversible factors. Similarly, findings from the Southern Community Cohort Study reported that hypertension and diabetes were stronger AF risk factors (as measured by HRs) in African Americans. ${ }^{37}$ More recently, findings from the Multiethnic Study of Atherosclerosis also showed that hypertension explained more of the population risk for $\mathrm{AF}$ among nonwhite participants. ${ }^{38}$

To explain the lower rates of $\mathrm{AF}$ in minority race-ethnic groups despite higher rates of traditional risk factors, we hypothesized that the hazards attributed to some of the traditional risk factors might be lower. However, we found that for the traditional cardiovascular risk factors except for age, the hazards were similar. It is possible that there are additional and important unmeasured environmental risks or other biologic factors that increase the risk of AF in NHWs or protect from AF in minority groups. Deeper characterization of risk factors will need to be performed in individual subgroups to identify these important, yet unidentified, characteristics.

Although the hazard from alcohol use was much higher in Asians compared with other race-ethnic groups, the $P$ for interaction was not significant after adjusting for multiple hypothesis testing. Nevertheless, if this finding can be validated in other cohorts, it may suggest that alcohol metabolites, which are higher in Asians who consume alcohol, ${ }^{39}$ might contribute significantly to AF risk. Mendelian randomization studies of variants in the alcohol dehydrogenase and acetaldehyde dehydrogenase genes may also be revealing.

Although existing AF prediction models perform well in African Americans, ${ }^{29,40}$ they have not been validated in other racial-ethnic cohorts. We found that the different commonly used AF predictive models perform equally well, if not better, among nonwhite women. This may be in part because the traditional risk factors included in these models account for a large proportion of the AR for AF among these groups. 
Table III. Performance of different AF prediction models as measured by c statistics with $95 \% \mathrm{Cls}$ for each race-ethnic group at 5 and 10 years

\begin{tabular}{|c|c|c|c|c|}
\hline Time & Race/Ethnicity & Model 1 & Model 2 & Model 3a \\
\hline \multirow[t]{5}{*}{$5 y$} & All & $0.80(0.79,0.80)$ & $0.80(0.79,0.80)$ & $0.79(0.78,0.79)$ \\
\hline & NHW & $0.79(0.79,0.80)$ & $0.80(0.79,0.80)$ & $0.79(0.78,0.79)$ \\
\hline & African American & $0.82(0.79,0.84)$ & $0.82(0.80,0.85)$ & $0.81(0.79,0.83)$ \\
\hline & Hispanic & $0.82(0.77,0.86)$ & $0.82(0.77,0.87)$ & $0.81(0.76,0.86)$ \\
\hline & Asian & $0.82(0.78,0.87)$ & $0.84(0.80,0.88)$ & $0.81(0.77,0.86)$ \\
\hline \multirow[t]{5}{*}{$10 y$} & All & $0.81(0.80,0.81)$ & $0.81(0.81,0.82)$ & $0.81(0.80,0.81)$ \\
\hline & $\mathrm{NHW}$ & $0.81(0.80,0.81)$ & $0.81(0.80,0.81)$ & $0.80(0.80,0.81)$ \\
\hline & African American & $0.83(0.82,0.85)$ & $0.84(0.82,0.85)$ & $0.83(0.81,0.85)$ \\
\hline & Hispanic & $0.85(0.82,0.87)$ & $0.85(0.82,0.87)$ & $0.84(0.81,0.87)$ \\
\hline & Asian & $0.87(0.83,0.90)$ & $0.87(0.84,0.90)$ & $0.85(0.82,0.89)$ \\
\hline Time & Race/Ethnicity & Model 3b & Model 4 & Model 5 \\
\hline \multirow[t]{5}{*}{$5 y$} & All & $0.79(0.78,0.80)$ & $0.80(0.79,0.80)$ & $0.78(0.77,0.78)$ \\
\hline & $\mathrm{NHW}$ & $0.79(0.78,0.80)$ & $0.79(0.79,0.80)$ & $0.77(0.77,0.78)$ \\
\hline & African American & $0.79(0.75,0.83)$ & $0.81(0.79,0.84)$ & $0.79(0.77,0.82)$ \\
\hline & Hispanic & $0.81(0.73,0.89)$ & $0.80(0.76,0.85)$ & $0.78(0.73,0.83)$ \\
\hline & Asian & $0.87(0.80,0.94)$ & $0.82(0.78,0.87)$ & $0.82(0.77,0.86)$ \\
\hline \multirow[t]{5}{*}{$10 y$} & All & $0.79(0.79,0.80)$ & $0.81(0.81,0.82)$ & $0.80(0.80,0.81)$ \\
\hline & NHW & $0.79(0.79,0.80)$ & $0.81(0.80,0.81)$ & $0.80(0.79,0.80)$ \\
\hline & African American & $0.79(0.76,0.82)$ & $0.83(0.82,0.85)$ & $0.82(0.80,0.84)$ \\
\hline & Hispanic & $0.79(0.74,0.84)$ & $0.84(0.81,0.87)$ & $0.83(0.80,0.86)$ \\
\hline & Asian & $0.82(0.75,0.88)$ & $0.86(0.82,0.89)$ & $0.85(0.82,0.89)$ \\
\hline
\end{tabular}

Model 1: primary model-age, educational status, BMl, hypertension, diabetes, hyperlipidemia, CAD, CHF, PAD, smoking, alcohol use. Model 2: primary model + physical activity, height. Model 3a: reduced Framingham model, does not include PR interval component. Model 3b: full Framingham model, ECG participants only ( $\mathrm{n}=50,428$ ). Model 4 : ARIC. Model 5: WHS. ${ }^{30}$

All WHI models are additionally adjusted for any fee-for-service CMS coverage during follow-up (yes/no) as well as for WHI intervention assignments

This study has several strengths including the large size and ethnic diversity in WHI and the deep characterization of the participants, which offers a unique opportunity to study the makeup of AF risk in different racial and ethnic cohorts. Potential limitations include errors in $\mathrm{AF}$ ascertainment by the use of ICD-9 codes, although the use of ICD-9 codes to identify incident $\mathrm{AF}$ has been validated in several large population studies, ${ }^{2,10,17}$ including the WHI. ${ }^{16}$ Because of the small number of incident atrial flutter cases, the study focused on incident AF but misclassification remains possible. For women with asymptomatic, paroxysmal AF, AF may be missed, resulting in misclassification, although this bias would be nondifferential by race and ethnicity and is a limitation in most long-term population studies that could only be overcome by continuous monitoring in the future. Finally, generalizability is limited because the study was conducted in postmenopausal women.

\section{Conclusions}

We found that although the incidence of $\mathrm{AF}$ is lower in minority groups, traditional risk factors account for a larger proportion of the AF burden in these groups. In addition, commonly used AF prediction models perform equally well and sometimes better in nonwhite groups. Future studies should explore novel genetic and environmental factors that explain the variance in AF risk among diverse populations.

\section{Acknowledgements}

A full listing of the WHI investigators can be found at: https://www.whi.org/researchers/Documents $\% 20 \% 20$ Write\%20a\%20Paper/WHI\%20Investigator\%20Long\%20List. pdf. We Thank the WHI investigators, staff, and study participants for their contributions.

\section{Disclosures}

The authors have no relationships with industry to disclose.

\section{References}

1. Go AS, Mozaffarian D, Roger VL, et al. Executive summary: heart disease and stroke statistics-2014 update: a report from the American Heart Association. Circulation 2014;129(3):399-410.

2. Go AS, Hylek EM, Phillips KA, et al. Prevalence of diagnosed atrial fibrillation in adults: national implications for rhythm management and stroke prevention: the AnTicoagulation and Risk Factors in Atrial Fibrillation (ATRIA) study. JAMA 2001;285:2370-5. [United States].

3. Wolf PA, Mitchell JB, Baker CS, et al. Impact of atrial fibrillation on mortality, stroke, and medical costs. Arch Intern Med 1998;158(3): 229-34. 
4. Benjamin EJ, Wolf PA, D'Agostino RB, et al. Impact of atrial fibrillation on the risk of death: the Framingham Heart Study. Circulation 1998;98(10):946-52

5. Soliman EZ, Safford $M M$, Muntner $P$, et al. Atrial fibrillation and the risk of myocardial infarction. JAMA Intern Med 2014;174(1):107-14.

6. Chen LY, Sotoodehnia N, Buzkova P, et al. Atrial fibrillation and the risk of sudden cardiac death: the Atherosclerosis Risk in Communities Study and cardiovascular health study. JAMA Intern Med 2013;173(1):29-35.

7. Feinberg WM, Blackshear JL, Laupacis A, et al. Prevalence, age distribution, and gender of patients with atrial fibrillation. Analysis and implications. Arch Intern Med 1995;155(5):469-73.

8. Friberg J, Scharling H, Gadsboll N, et al. Comparison of the impact of atrial fibrillation on the risk of stroke and cardiovascular death in women versus men (The Copenhagen City Heart Study). Am J Cardiol 2004;94:889-94. [United States].

9. Benjamin EJ, Levy D, Vaziri SM, et al. Independent risk factors for atrial fibrillation in a population-based cohort. The Framingham Heart Study. JAMA 1994;271(11):840-4.

10. Psaty BM, Manolio TA, Kuller LH, et al. Incidence of and risk factors for atrial fibrillation in older adults. Circulation 1997;96(7):2455-61.

11. Stewart S, Hart CL, Hole DJ, et al. Population prevalence, incidence, and predictors of atrial fibrillation in the Renfrew/Paisley study. Heart 2001;86(5):516-21.

12. Krahn AD, Manfreda J, Tate RB, et al. The natural history of atrial fibrillation: incidence, risk factors, and prognosis in the Manitoba Follow-Up Study. Am J Med 1995;98(5):476-84.

13. Bush D, Martin LW, Leman R, et al. Atrial fibrillation among African Americans, Hispanics and Caucasians: clinical features and outcomes from the AFFIRM trial. J Natl Med Assoc 2006;98(3):330-9.

14. Ball J, Carrington MJ, McMurray JJ, et al. Atrial fibrillation: Profile and burden of an evolving epidemic in the 21 st century. Int J Cardiol 2013;167:1807-24.

15. Dewland TA, Olgin JE, Vittinghoff $E$, et al. Incident atrial fibrillation among Asians, Hispanics, blacks, and whites. Circulation 2013;128(23):2470-7.

16. Perez MV, Wang PJ, Larson JC, et al. Risk factors for atrial fibrillation and their population burden in postmenopausal women: the Women's Health Initiative Observational Study. Heart 2013;99(16):1173-8.

17. Alonso A, Agarwal SK, Soliman EZ, et al. Incidence of atrial fibrillation in whites and African-Americans: the Atherosclerosis Risk in Communities (ARIC) study. Am Heart J 2009;158(1):111-7.

18. Borzecki AM, Bridgers DK, Liebschutz JM, et al. Racial differences in the prevalence of atrial fibrillation among males. J Natl Med Assoc 2008; 100(2):237-45.

19. Perez MV, Yaw TS, Myers J, et al. Prognostic value of the computerized ECG in Hispanics. Clin Cardiol 2007;30(4):189-94.

20. Marcus GM, Alonso A, Peralta CA, et al. European ancestry as a risk factor for atrial fibrillation in African Americans. Circulation 2010;122(20):2009-15.

21. Perez MV, Hoffmann TJ, Tang H, et al. African American race but not genome-wide ancestry is negatively associated with atrial fibrillation among postmenopausal women in the Women's Health Initiative. Am Heart J 2013;166(3):566-72.

22. Design of the Women's Health Initiative clinical trial and observational study. The Women's Health Initiative Study Group. Control Clin Trials 1998;19(1):61-109.

23. Langer RD, White $E$, Lewis $C E$, et al. The Women's Health Initiative Observational Study: baseline characteristics of participants and reliability of baseline measures. Ann Epidemiol 2003;13(9 Suppl): S107-21.

24. Curb JD, McTiernan A, Heckbert SR, et al. Outcomes ascertainment and adjudication methods in the Women's Health Initiative. Ann Epidemiol 2003;13(9 Suppl):S122-8.

25. Azarbal F, Stefanick ML, Salmoirago-Blotcher E, et al. Obesity, physical activity, and their interaction in incident atrial fibrillation in postmenopausal women. J Am Heart Assoc 2014;3(4):1-9.

26. Johnson-Kozlow M, Rock CL, Gilpin EA, et al. Validation of the WHI brief physical activity questionnaire among women diagnosed with breast cancer. Am J Health Behav 2007;31(2):193-202.

27. Perez MV, Wang PJ, Larson JC, et al. Effects of postmenopausal hormone therapy on incident atrial fibrillation: the Women's Health Initiative randomized controlled trials. Circ Arrhythm Electrophysiol 2012;5(6):1108-16.

28. Schnabel RB, Sullivan LM, Levy D, et al. Development of a risk score for atrial fibrillation (Framingham Heart Study): a community-based cohort study. Lancet 2009;373(9665):739-45.

29. Chamberlain AM, Agarwal SK, Folsom AR, et al. A clinical risk score for atrial fibrillation in a biracial prospective cohort (from the Atherosclerosis Risk in Communities [ARIC] study). Am J Cardiol $2011 ; 107(1): 85-91$.

30. Everett $B M$, Cook NR, Conen $D$, et al. Novel genetic markers improve measures of atrial fibrillation risk prediction. Eur Heart J 2013;34(29):2243-51.

31. Naderi S, Rodriguez F, Wang Y, et al. Racial disparities in hospitalizations, procedural treatments and mortality of patients hospitalized with atrial fibrillation. Ethn Dis 2014;24(2):144-9.

32. Soliman EZ, Alonso A, Goff Jr DC. Atrial fibrillation and ethnicity: the known, the unknown and the paradox. Future Cardiol 2009;5(6): 547-56.

33. Haywood $\sqcup$, Ford CE, Crow RS, et al. Atrial fibrillation at baseline and during follow-up in ALLHAT (Antihypertensive and Lipid-Lowering Treatment to Prevent Heart Attack Trial). J Am Coll Cardiol 2009;54(22):2023-31.

34. Upshaw Jr CB. Reduced prevalence of atrial fibrillation in black patients compared with white patients attending an urban hospital: an electrocardiographic study. J Natl Med Assoc 2002;94(4):204-8.

35. Thomas KL, Piccini JP, Liang $L$, et al. Racial differences in the prevalence and outcomes of atrial fibrillation among patients hospitalized with heart failure. J Am Heart Assoc 2013;2(5): e000200. [1-10].

36. Lau CP, Gbadebo TD, Connolly SJ, et al. Ethnic differences in atrial fibrillation identified using implanted cardiac devices. J Cardiovasc Electrophysiol 2013;24(4):381-7.

37. Lipworth L, Okafor H, Mumma MT, et al. Race-specific impact of atrial fibrillation risk factors in blacks and whites in the southern community cohort study. Am J Cardiol 2012;110(11):1637-42.

38. Rodriguez CJ, Soliman EZ, Alonso A, et al. Atrial fibrillation incidence and risk factors in relation to race-ethnicity and the population attributable fraction of atrial fibrillation risk factors: the Multi-Ethnic Study of Atherosclerosis. Ann Epidemiol 2015;25(2):71-6. [76 e71].

39. Suddendorf RF. Research on alcohol metabolism among Asians and its implications for understanding causes of alcoholism. Public Health Rep 1989;104(6):615-20.

40. Alonso A, Krijthe BP, Aspelund T, et al. Simple risk model predicts incidence of atrial fibrillation in a racially and geographically diverse population: the CHARGE-AF consortium. J Am Heart Assoc 2013;2(2):e000102. [1-11]. 